on doit toujours avoir $n_{1}>0$. Cette condition est satisfaite sur le parement amont $\left(x=0, n_{1}=y\right)$. On vérifiera (p'elle l'est aussi sur le parement aval pour $x=e$.

La compression maxima en un point quelconque, c'est-àdíre la pression exercée sur la ligne isostatique $(A)$ passant en ce point, est:

$$
A=\frac{n+n_{1}}{2}+\frac{1}{2} \sqrt{\left(n-n_{1}\right)^{2}+4 t^{2}}
$$

Si $A$ est une fonction croissante de $x$, son maximum a lieu sur le parement aval et il est facile de vérifier que son expression coïncide avec (12). Si $A$ devenait maximum par une valeur de $x$ comprise entre $x=0$ et $x=e$, c'est cette valeur de $A$ qu'il faudrait faire inférieure à la limite $\rho$ qu'on s'est imposé pour la résistance des maçonneries à la compression. Pour $x=0$ on a $t=0$ et $A=n=n$ '.

La compression minima $B$ a pour expression:

$$
B=\frac{n+n_{1}}{2}-\frac{1}{2} \sqrt{\left(n-n_{1}\right)^{2}+4 t^{2}}
$$

et l'on doit avoir partout $B \geqslant 0$. Au parement aval $B=0$; cette condition est remplie sur le parement amont car on $y$ a: $B=n_{1}=y$. Elle l'est également partout si $B$ est une lonction décroissante de $x$.

La force tangentielle maxima en un point donné a pour expression:

$$
C=\frac{A-B}{2}=\frac{1}{2} \sqrt{\left(n-n_{1}\right)^{2}+4 t^{2}}
$$

sur le parement aval où $B=0$, on a $C=\frac{A}{2}$ ainsi que nous lavons vu précédemment.

Si l'on représente par $n_{\mathrm{v}}$ la composante normale et $n_{\mathrm{T}}$ la composante tangentielle de la pression totale exercée sur un joint oblique quelconque, la force produisant le cisaillement sera $n_{\mathrm{r}}-f n_{\mathrm{v}}, f$ étant le coefficient de frottement. Son maximum a pour expression:

$$
\frac{\sqrt{1+f^{2}} \sqrt{\left(n-n_{1}\right)^{2}+4 t^{2}}-f\left(n+n_{1}\right)}{2}
$$

de telle sorte qu'en tenant compte à la fois de la résistance au frottement et de la résistance au cisaillement, on doit aroir :

$$
\frac{1}{2} \sqrt{\left(n-n_{1}\right)^{2}+4 t^{2}} \leqslant \frac{1}{\sqrt{1+f^{2}}}\left[\rho^{\prime}+f \frac{\left(n+n_{1}\right)}{2}\right]
$$

f'étant la résistance pratique de la maçonnerie au cisaillement.

Les expressions de $A, B, C$ resteraient les mêmes si le parement amont était incliné, mais les équations de $n^{\prime}, n, n, n_{1}$ el $t$ seraient plus compliquées.

(A suivre)

\section{H. BELlet.}

\section{LE REBOISEMENT EN AUVERGNE}

Un grand nombre de landes et de terres incultes se prêteraient au reboisement dans la région du Centre, surtout dans les régions montagneuses d'Aubrac, de la Viadine, du Lévezou et sur les plateaux du Causse et du Ségala ; cette améloration, si elle se réalisait partout où elle est possible, aurait pour résultats, non seulement d'augmenter la valeur des terrains reboisés, mais encore d'exercer une heureuse influence lur le climat de la région. Mon intention n'est pas de développer ces idées qui sont acceptées de tout le monde, mais de faire connaître les plus pratiques et les plus économiques, de réaliser des plantations avec le concours de pépinières et sécheries de l'administration des forêts.

L'administration des forêts entretient, dans le département du Cantal, une pépinière à Arpajon et une sécherie à Murat, destinées à fournir aux communes et aux particuliers des plants et des graines pour favoriser l'œuvre du reboisement. des montagnes. Ces deux élablissements, ne desservent pas seulement le Cantal, les plants et les grannes peuvent être expédiés partout. Les renseignements suivants sont destinés à faciliter au public les moyens d'en tirer tous les avantages qu'ils ont pour but de lui offrir.

On cultive actuellement dans la pépinière d'Arpajon, le Pin sylvestre d'Auvergne, l'Epicéa, le Sapın, le Mélèze, le Pin noir, le Chêne, le Châtaignier et le Robinier faux Acacia. Le Pin d'Auvergne et l'Epicéa, considérés comme les essences les mieux appropriées aux grands travaux de reboisement de la région, occupent dans la pépinière le rang principal, et ont, jusqu'à présent, pu être fournis en quantité illimitée. La culture des autres essences, moins employées, est par conséquent plus restreinte.

L'administration des forêts délivre les plants de la Pépinière d'Arpajon, d'après deux modes différents, soit à prix d'argent, soit à titre de subvention.

Dans le premier cas (délivrance à prix d'argent), les plants sont délivrés aux prix et conditions fixés par un tarif arrêté, chaque année, par le conservateur et qui est adressé à toute personne en faisant la demande. Ce tarif donne, sur l'emballage et le transport des plants, de même que sur les formalités à remplir pour en obtenir la livraison, tous les renseignements nécessaires.

Aucune justification n'est exigée pour la délivrance des plants à prix d'argent. La vente en est consentie à toute personne qui en sollicite la concession.

Toute demande de plants doit être adressée à M. l'Inspecteur des eaux et forêts à Aurillac (Cantal), et doit être accompagnée d'un timbre-poste pour l'affranchissement de la réponse.

Cette réponse fait connaître si les ressources disponibles de la pépinière permettent la livraison des plants demandés.

Le pétitionnaire doit, au vu de la réponse, si elle est affurmative, se présenter cinq jours après sa réception, au bureau du receveur des domaines du chef-lieu de canton dont dépend sa résidence, pour verser la somme indiquée dans la dite lettre.

Immédiatement après, l'intéressé doit adresser la quittance à l'inspecteur des eaux et forêts à Aurillac et lui faire connaître l'époque à laquelle il désire recevoir les plants, l'adresse exacte du destinataire, la gare à laquelle il y a lieu de les expédier et le mode de transport à employer (grande ou petite vitesse).

L'envoi des plantes n'a lieu qu'après l'accomplissement de ces formalités, la justification du paiement devra précéder toute livraison.

Si cette justification n'est pas faite dans les quinze jours de la date de la lettre d'avis, la demande est considérée comme non avenue.

Dans les pays de montagnes, des subventions peuvent être accordées aux communes, aux établissements publics et aux particuliers, à raison des travaux entrepris par eux pour l'amélioration, la consolidation $\mathrm{du}$ sol et la mise en valeur des pâturages (article 5 de la loi du 4 avril 1882).

Dans la région, ces subventions consistent en la délivrance de graines ou de plants.

Les demandes doivent être rédigées sur des formules spéciales fournies par l'administration des forêts. Les propriétaires aui désirent prendre part aux subventions accordées par l'Etat doivent s'adresser, d'abord, à l'agent forestier le plus voisin (l'inspecteur ou le sous-inspecteur des forêts dans chaque dénartement), pour obtenir les formules nécessaires. Celles-ci, dîment remnlies et timbrées, sont transmises : $\mathrm{I}^{\circ}$ S'il s'agit d'un particulier, au conservateur des forêts dans la circonscription duquel se trouve la propriété à reboiser ; 
$2^{\circ}$ S'il s'agit d'une commune ou d'un établissement public, au préfet du département, qui la transmet au conservateur avec son avis motivé

A ces renseignements empruntés aux notices de l'Admmistration des Forêts, j'ajouteraı que 5.000 plants sont nécessaires pour la plantation d'un hectare, et 5 kilogrammes de graines de pin pour le semis d'un hectare ; que le pin sylvestre d'Auvergne convient plus spécralement aux terrains siliceux, le pin noir d'Autriche aux terrains calcarres, les sapıns et les épicéas à des terrains froıds et humides à de très hautes altitudes

11 me reste maintenant à explıquer dans quelles conditions doivent être farts les plantations et les semis pour donner les mellleurs résultats.

Le sol à planter peut être nu ou bien envahı par des genêts, bruyères, ajoncs, fougères, ronces, etc. Dans ce dernier cas, il convient de le débarrasser tout d'abord de ces plantes adventices. On peut procéder à cette opération, soit d'une façon complète en faisant un écobuage préalable, soit partıculièrement en nettoyant l'emplacement que devront occuper les plants sur une surface de I mètre carré, ou encore en divisant le terrain par handec alternées transversales à la pente, distantes de I m. 50 et en dégageant à la bêche, sur chacune de ces bandes, un espace de $0 \mathrm{~m}$. 50, dans lequel on procédera ensuite aux semis ou plantations laissant en firche les intervalles de I mètre compris entre chaque bande

Le premier procédé, qui consiste à débarrasser complètement le sol des plantes adventices, est préférable, car les jeunes arbres ne risquent pas ainsi d'être étouffés par cette végétation spontanée

Si on adopte ce système, on peut utılement tracer des raies à la charrue à I m. 50 l'une de l'autre pour marquer les lignes d'arbres Il importe de diriger ces raies aussi horizontalement que possible, comme si l'on suivait une ligne de niveau. Il faut avoir soin de retourner, sur le bord inférieur, la bande détachée par la charrue; par conséquent, dans les terrains en pente, on travaille à raie perdue, à moins qu'on ne se serve d'une charrue tourne-oreille C'est dans ces espèces de rigoles à niveau que viennent s'accumuler les débris végétaux et les terres entraînées par les pluies, pour favoriser la croissance du plant.

Pour tracer ces raies de charrues, distantes de $1 \mathrm{~m}$. 50, voici comment on opère : le laboureur jalonne préalablement, de distance en distance, la ligne du niveau qu'il devra suivre puis 1 l se dirige, avec la charrue, vers les piquets ainsi plantés. Arrivé au premier piquet, il l'arrache et le plante à côté, à la distance voulue ( $1 \mathrm{~m}$. 50). Arrivé au second, il opère de même, et ainsi de suite, il avance en jalonnant la ligne suivante qu'il aura à parcourir. On arrive de la sorte, à tracer, par hectare, 6600 mètres de bandes distantes de I m. 50 ; un laboureur ordinaıre trace aisément au moins un hectare par jour.

Dans les terrains non préparés, la réussite de la plantation ou du semis est presque assurée et l'opération elle-même fort expéditive Un ouvrier armé d'une bêche suit les lignes trarées et plante un jeune pred on sème en poquets deux ou trois graines tous les I m. 25 ou I m. 50. Il peut facilement planter I oon pieds par jour Chaque pied doit être enterré jusqu'au collet et convenablement tassé pour lutter contre la sécheresse et contre le déchaussement produit par les gelées.

$S_{1}$ c'est d'un semis qu'il s'agit, on peut également semer les graines à la volée et les enfouir par un coup de herse.

Dès le début, il importe, au premier chef, d'enfermer le terrain à reboiser, de le défendre par une clôture respectable, fossć, mur ou banquette de terre, palissade, haies ou ronces artificielles. C'est l'homme ou les animaux qu'il faut empêrher d'entrer Le bétail, vollà l'ennemi. Coupez une lande en deux par une barrière infranchissable ; d'un côté laissez pâ̂tre el de l'autre mettez en défens; venez-y voir dix ans après, vous ne reconnaîtrez plus le terrain clos

Il n'est pas d'exemple plus probant que celui de la Cour des Comptes, transformée en une forêt vierge après vingthuit ans d'inoccupation.

Il faut, dans la. suite, procéder tous les dix ou quinze ans, à des éclaircies périodiques représentant environ $\mathrm{I} / 10^{\circ}$ des arbres occupant. le sol.

(I.a Revue Industruelle.)

E. MARRE,

Professeur départemental d'A griculture de l'Aveyron.

\section{LE MOIS HYDRO-ÉLECTRIQUE}

\section{ACADF́MIE DES SCIENCES}

\section{MÉGANIQUE ET ÉLEGTRIGITÉ}

Sur un nouvel embrayage. - Note de MM. le DUC DE Gurche et $\mathrm{H}$. Gilardoni.

Considérons (fig. 1) un tambour $T$ de centre $O$, à l'intérieur duquel frotte un patin $p$ relié au centre $O$ par les deux bielles $0 A$ et $O B$; pour un chorx convenable de l'angle $\sigma$ de $O B$ et de $A B$, l'appareil constitue un embrayage grâce auquel le patin $p$ est entrainé, quelle que soit la résistance à vaincre.

Désignons en effet par $S$ la composante normaie de la bielle $A B$ sur le patin, et par $P$ la force tangentielle d'entraînement du tambour; le patin, par la liaison $\mathrm{OAB}$, est assujetti à tourner autour de $\mathrm{O}$; la somme des moments des forces qui agissent sur lui par rapport à $\mathrm{O}$ est donc nulle, et l'on a, en négligeant les frottements aux axes:

$$
(a-b) S \operatorname{tg} \sigma=P a
$$

en désignant par $a$ le rayon du tambour et par $b$ la distance du point $B$ au tambour. Or, pour que le patin ne glisse pas, il faut que :

$$
P \leqslant S f \quad \text { d'où } \quad \operatorname{tg} \sigma \leqslant \frac{a f}{a-b}
$$

condition indépendante de $S$; donc, dès que $\sigma$ remplira la condition (I) il y aura entrainement, quelle que soit la pression $S$.

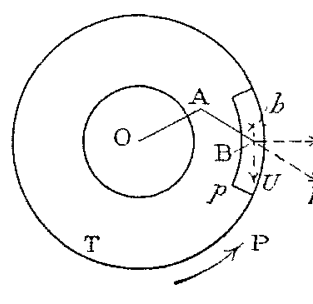

Fig. 1 .

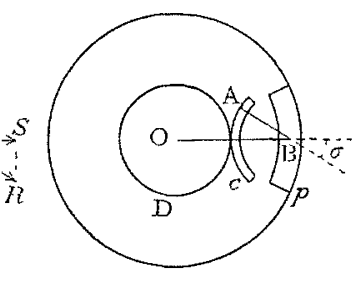

Fig. 2.

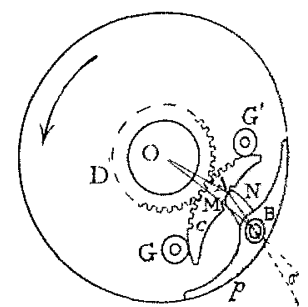

Fig. 3.
Ce dispositif bien connu n'est pas progressif, car, la valeur de o étant parfaitement déterminée par les dimensions de l'appareil, il $y$ a coincement dès que le patin touche le tambour. Une modification, permettant de faire varier $\sigma$, rend l'appareil progressif. Lorsque $\sigma$ sera plus grand que l'angle limite $\varphi$, il y aura glissement du patin avec entrainement partiel (ou freinage), et l'entrainement sera complet lorsque $\sigma$ tombera au-dessous de la valeur $\phi$.

Pour atteindre ce but, nous supprimons la bielle OA (fig. 2); la bielle $\mathrm{AB}$ est articulée sur un secteur circulaire $\mathrm{C}$, guidé et astreintà décrire un cercle de centre $B$. Pour une position déterminée du secteur $C$, et dès qu'il y aura contact, tout se passera comme si $O$ et $A$ étaient reliés par une bielle.

Ces résultats sont exacts tant qu'il n'y a ni usure ni déformation des pièces en contact, car ces deux causes concourent à dimınuer la valeur de l'angle $\sigma$ qui correspond à une position déterminée du secteur. Pour éviter cet inconvénient, on peut remplacer le pivot $A$ par deux courbes tangentes $M$ et $N$ (fig. 3 ), liées, I'une à l'axe 0 , l'autre au cliquet B. Si M est un cercle décrit de O comme centre et $N$ une développante d'un cercle de centre $B$, les valeurs de l'angle $A O B$ et de l'angle de la normale commune en $A$ avec la force BA ne sont pas modifiées par l'usure. L'invariabilité de l'angle de la normale en A évite les glissements relatits de $M$ et $N$.

Le secteur $C$ est guidé par deux galets $G$ et $G$ ' dont les centres se trouvent sur deux rayons à angle drolt (fig. 3): quatre galets permettent ainsi de guider quatre secteurs tels que $C$. Ces secteurs portent des dents qui engrènent sur une roue dentée de centre $0 . L$ rotation de celle-ci comnande donc tout le système d'embrayage. 